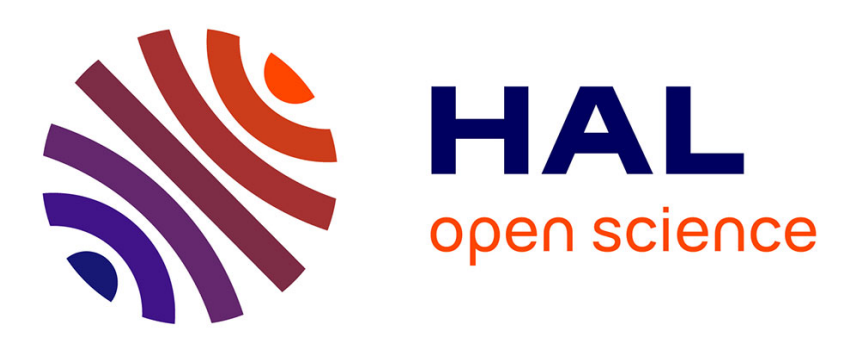

\title{
Nonlinear observers in vision system: Application to civil aircraft landing
}

Victor Gibert, Laurent Burlion, Abdelhamid Chriette, Josep Boada, Franck

Plestan

\section{- To cite this version:}

Victor Gibert, Laurent Burlion, Abdelhamid Chriette, Josep Boada, Franck Plestan. Nonlinear observers in vision system: Application to civil aircraft landing. 14th European Control Conference (ECC'15), Jul 2015, Linz, Austria. pp.1818-1823, 10.1109/ECC.2015.7330802 hal-01317569

\section{HAL Id: hal-01317569 \\ https://hal.science/hal-01317569}

Submitted on 18 May 2016

HAL is a multi-disciplinary open access archive for the deposit and dissemination of scientific research documents, whether they are published or not. The documents may come from teaching and research institutions in France or abroad, or from public or private research centers.
L'archive ouverte pluridisciplinaire HAL, est destinée au dépôt et à la diffusion de documents scientifiques de niveau recherche, publiés ou non, émanant des établissements d'enseignement et de recherche français ou étrangers, des laboratoires publics ou privés. 


\title{
Nonlinear observers in vision system : Application to civil aircraft landing
}

\author{
Victor GIBERT ${ }^{\star}$, , Laurent BURLION ${ }^{\diamond}$, Abdelhamid CHRIETTE ${ }^{\dagger}$, Josep BOADA ${ }^{\star}$ and Franck PLESTAN ${ }^{\dagger}$
}

\begin{abstract}
In this paper, a new pose estimation solution for perspective vision system is presented and applied to a civil aircraft landing phase. Vision sensor is used to overcome the need for external technologies and runway knowledge. Two nonlinear observers are proposed for on-line estimation of the deviations of the aircraft w.r.t. the runway. These nonlinear observers are based, first, on a high-gain approach, and then on a high-order sliding-mode approach, allowing robustness and finite time convergence. The originality of the presented results consists in estimating deviations with redundant informations in order both to increase efficiency of the observer and guarantee observability. Simulation results obtained on a realistic landing scenario are presented.
\end{abstract}

\section{INTRODUCTION}

Today, civil aircrafts land automatically thanks to external technology. The most common used system, named ILS (Instrument Landing System) allows aircrafts to land without pilot action (except monitoring). Other solutions for localization are Differential GPS, IRS ( Inertial Reference System) or VOR/DME (VHF Omnidirectional Range/Distance Measuring Equipment). These technologies are not available everywhere (unequipped or unknown airport) and everytime (probability of failure). To cope with these issues (availability of an accurate absolute position) and extend automatic landing coverage, the use of cameras as additional sources of information, is going to be investigated. In the last ten years, camera technology has made a technological leap so that equipping every aircraft seems to be easy and cheep.

Visual servoing consists in using a vision sensor and computer vision algorithms in order to control the motion of the system (see tutorial in [1]). The first class of control, named PBVS (Pose Based Visual Servoing), consists in using visual measurements in order to estimate deviation or orientation of the camera. The second class of control, named IBVS (Image based Visual Servoing), consists in controlling the coordinates of visual features in the image plane. IBVS solutions applied to the automatic landing of an aircraft have been studied for the last decade; in [2][3][4][5][6], are propose guidance solutions in order to reach and track the desired approach trajectory. Nevertheless, these scheme needs to develop new guidance laws with a complete chain (composed by image capture, image processing and nonlinear guidance algorithms) which might be difficult to certify

${ }^{\star}$ Airbus Operations S.A.S., Toulouse, France. E-mail: victor.gibert@airbus.com, josep.boada-bauxell@airbus.com.

${ }^{\circ}$ ONERA, the French Aerospace Lab, 31055, Toulouse, France. E-mail: laurent.burlion@onera.fr.

†LUNAM Université, Ecole Centrale de Nantes-IRCCyN, Nantes, France E-mail: Abdelhamid.Chriette@irccyn.ec-nantes.fr, Franck.Plestan@irccyn.ec-nantes.fr. by the authorities.

The PBVS scheme could be divided in two steps, the first one estimates the deviations of the aircraft w.r.t. the runway. Then, in a second step, these deviations are used in certified guidance laws. Most of the time, vision sensor provides a 2D view of the outside world. Then, an unknown scale factor prevents the system to directly measure the $3 \mathrm{D}$ deviations. In this purpose, observer needs to be used.

Estimation of the 3D deviations can be obtained by using more than one camera [7]. Nevertheless, stereo-vision appears difficult in civil aircraft application because the distance from runway is important, thus, calibration must be very precise. An other solution is based on knowledge of seen objects dimensions [8]. However, a generic runway is considered whose size and markers are not known: geometric reconstruction solutions using these informations can not be applied. The last solution consists in using a monocular camera and taking into account its motions [9][10][11]. In civil aircraft applications, the motion of the aircraft could be considered known, hence the use of dynamics of visual features between several images is sufficient to estimate deviations w.r.t. the runway. This solution is selected even if previous studies in [9]-[11] have not been applied to a landing scenario and only used one single point for estimation.

Hence, there is a real interest to develop estimation based solutions in order to estimate the deviations of the camera w.r.t. to the runway. In this purpose, the single available informations are the knowledge on rotational and translational velocities provided by IRS (Inertial Reference System) and visual measurements. The visual informations, provided by image processing algorithms, correspond to the perspective projection of the $3 \mathrm{D}$ corners of the runway in the image plane.

The main contribution of this paper is to propose observation solutions based on nonlinear approach that ensure performance despite the potential loss of observability and provide robustness because of redundancy of the information.

The paper is organized as follows. In Section II, problem formulation and context of the study are presented. Section III presents nonlinear observation solutions. Afterwards, the application of the presented observers to civil aircraft landing scenarios is proposed in Section IV. Finally, Section V concludes this paper.

\section{Problem Statement}

In this paper, the problem under interest consists in using a monocular camera embedded on a civil aircraft in order to 
overcome the need of external systems during an automatic landing. The single useful informations come from IRS and camera, IRS providing orientation and velocities whereas camera is providing visual features obtained from Image Processing. The main goal is to estimate deviations of the aircraft w.r.t. the runway.

In final approach, the desired trajectory, named glide path, is ending on the runway at a point $\mathbf{E}$ (see Figure 1). The missing informations which need to be estimated are the unmeasured 3D deviations $\Delta_{X}, \Delta_{Y}$ and $\Delta_{H}$ of the aircraft w.r.t. E, expressed in the inertial frame $\left(\mathscr{F}_{i}\right)$. Then, dynamics
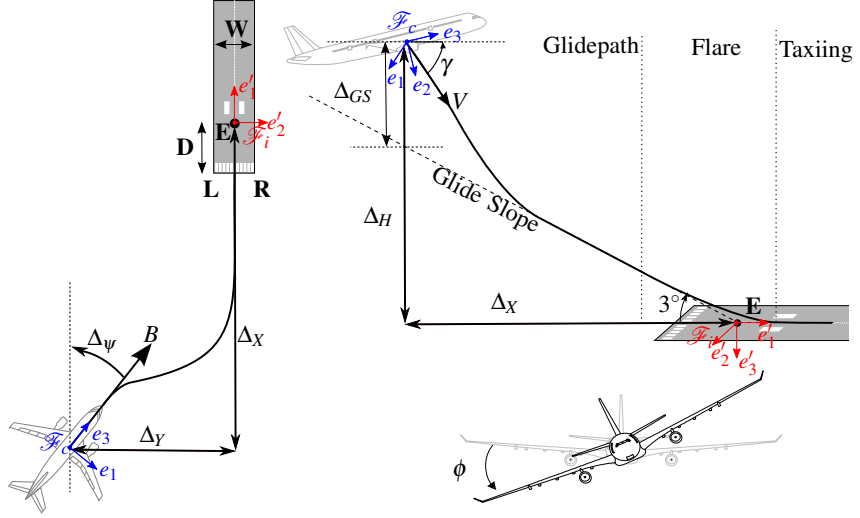

Fig. 1: Notations used in landing phase.

of $\left[\begin{array}{lll}\Delta_{X} & \Delta_{Y} & \Delta_{H}\end{array}\right]^{T}$ reads as

$$
\begin{aligned}
& \dot{\Delta_{X}}=V_{X}(t) \\
& \dot{\Delta_{Y}}=V_{Y}(t) \\
& \dot{\Delta_{H}}=V_{H}(t)
\end{aligned}
$$

with $V_{i}$ the known translational velocities expressed in the inertial frame $\left(\mathscr{F}_{i}\right)$.

The choice of the visual features is a critical point to guarantee observability. Instead of using a single point, which could lead to loss of observability, in this paper, the visual features are based on 2 points $\mathbf{L}$ and $\mathbf{R}$ which are the first two corners of the runway (see Figure 1). This particular choice allows ensuring observability during all the final approach phases. From visual features $\Theta_{L}, d_{y_{L}}, \Theta_{R}$ and $d_{y_{R}}$ presented in Figure 2, a measurement vector $\mathbf{y}$ is obtained and directly linked with the 3D deviations between the aircraft and points $\mathbf{L}\left(\left[\Delta_{X_{L}} \Delta_{Y_{L}} \Delta_{H_{L}}\right]^{T}\right)$ and $\mathbf{R}\left(\left[\Delta_{X_{R}} \Delta_{Y_{R}} \Delta_{H_{R}}\right]^{T}\right)$. It gives

$$
\begin{gathered}
y_{1_{L}}=\frac{\Delta_{X_{L}}}{\Delta_{H_{L}}}=g_{1}\left(\Theta_{L}, d_{y_{L}}\right) \\
y_{2_{L}}=\frac{\Delta_{Y_{L}}}{\Delta_{H_{L}}}=g_{2}\left(\Theta_{L}, d_{y_{L}}\right) \\
y_{1_{R}}=\frac{\Delta_{X_{R}}}{\Delta_{H_{R}}}=g_{1}\left(\Theta_{R}, d_{y_{R}}\right) \\
y_{2_{R}}=\frac{\Delta_{Y_{R}}}{\Delta_{H_{R}}}=g_{2}\left(\Theta_{R}, d_{y_{R}}\right) .
\end{gathered}
$$

The functions $g_{1}(\cdot)$ and $g_{2}(\cdot)$, derived from [5], theoretically represent a combination of the deviations $\Delta_{X}, \Delta_{Y}$ and $\Delta_{H}$ expressed in the inertial frame. Moreover, $\mathbf{L}$ and $\mathbf{R}$ have the

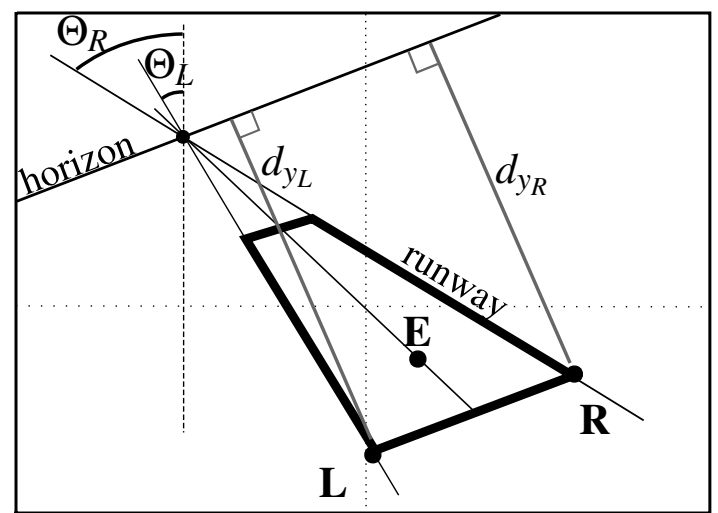

Fig. 2: Visual features $\Theta$ and $d_{y}$ in the image plane: the bold quadrilateral is representing the runway.

same values for $\Delta_{X}$ and $\Delta_{H}$. Then, $\mathbf{y}$ can be expressed as a function of the variables $\Delta_{X}, \Delta_{Y}$ and $\Delta_{H}$ as

$$
\boldsymbol{y}=\left[\begin{array}{l}
y_{1} \\
y_{2} \\
y_{3}
\end{array}\right]=\varepsilon\left[\begin{array}{lll}
\frac{\Delta_{X}-D}{\Delta_{H}} & \frac{\Delta_{Y}-\frac{W}{2}}{\Delta_{H}} & \frac{\Delta_{Y}+\frac{W}{2}}{\Delta_{H}}
\end{array}\right]^{T}
$$

with $\varepsilon$ the focal length of the camera, $W$ the unknown runway width and a known fixed distance $D=320 \mathrm{~m}$ between the beginning of the runway and the glide path ending point $\mathbf{E}$. Note that $y_{1}$ is given by $y_{1_{L}}=y_{1_{R}}, y_{2}$ by $y_{2_{L}}$ and $y_{3}$ by $y_{2_{R}}$.

Remark 1: The measurement vector (3) only gives the measurement of $y_{1}, y_{2}$ and $y_{3}$ but $\Delta_{X}, \Delta_{Y}, \Delta_{H}$ and $W$ are unknown. The objective is to propose a solution in order to estimate the variables $\Delta_{X}, \Delta_{Y}$ and $\Delta_{H}$. In the sequel, it is shown that estimation of $W$ is trivial but not needed in this paper.

In order to simplify the problem, as used in [12], consider the new state vector

$$
z=\left[\begin{array}{l}
z_{1} \\
z_{2} \\
z_{3} \\
z_{4}
\end{array}\right]=\left[\begin{array}{l}
y_{1} \\
y_{2} \\
y_{3} \\
\frac{1}{\Delta_{H}}
\end{array}\right]
$$

with $\left[\begin{array}{lll}z_{1} & z_{2} & z_{3}\end{array}\right]^{T}$ the measured states variables and $z_{4}=\frac{1}{\Delta_{H}}$ the unmeasurable variable which will be estimated. Then, system (1)-(3) reads as $\dot{z}=f(z)$ with

$$
\begin{aligned}
& \dot{z}_{1}=z_{4}\left(V_{X}-V_{H} z_{1}\right) \\
& \dot{z}_{2}=z_{4}\left(V_{Y}-V_{H} z_{2}\right) \\
& \dot{z}_{3}=z_{4}\left(V_{Y}-V_{H} z_{3}\right) \\
& \dot{z}_{4}=-V_{H} z_{4}^{2} \\
& y=\left[\begin{array}{lll}
z_{1} & z_{2} & z_{3}
\end{array}\right]^{T} .
\end{aligned}
$$

The estimation objective can be summarized as follows

- design an observer for (5) giving an estimation of $z_{4}$, i.e. of $\Delta_{H}$, denoted $\hat{\Delta}_{H}$; 
- from the estimation $\hat{\Delta}_{H}$ and the knowledge of the focal length $\varepsilon$ and distance $D$, an estimation of $\Delta_{X}$, denoted $\hat{\Delta}_{X}$, is derived from

$$
\hat{\Delta}_{X}=\frac{\hat{\Delta}_{H} \cdot z_{1}}{\varepsilon}+D
$$

- from the estimation $\hat{\Delta}_{H}$, the knowledge of the focal $\varepsilon$ and given that $\Delta_{Y}=\frac{1}{2}\left(\Delta_{Y_{L}}+\Delta_{Y_{R}}\right)$, an estimation of $\Delta_{Y}$, denoted $\hat{\Delta}_{Y}$, is derived from

$$
\hat{\Delta}_{Y}=\frac{\hat{\Delta}_{H} \cdot\left(z_{2}+z_{3}\right)}{2 \varepsilon}
$$

Note that the observer solution described in the sequel gives also estimations of the measurements $\left(\hat{z}_{1}, \hat{z}_{2}\right.$ and $\left.\hat{z}_{3}\right)$. Such estimations can be used in order to compute both evaluations $\hat{\Delta}_{X}$ and $\hat{\Delta}_{Y}$. This is interesting to use measurement estimations because observer is acting as a filter, reducing the noise. Note also that the width of the runway can be computed from estimated deviations by $W=2\left(\hat{\Delta}_{Y}-y_{3} \hat{\Delta_{H}}\right)$.

\section{OBSERVER DESIGN FOR OVERSIZED SYSTEM}

The proposed observer design for system (5) is based on a canonical form, which allows an easier design. This Section shows how to manage the observability singularities.

\section{A. Observability analysis}

Following the standard way to evaluate the observability feature of (5), consider the transformation

$$
\Psi=\left[\begin{array}{llll}
z_{1} & z_{2} & z_{3} & \dot{z}_{1}
\end{array}\right]
$$

In this case, the so-called observability indicies [13] are (if system (5) is observable) respectively for $y_{1}, y_{2}, y_{3}[2,1,1]$. Then, the observability condition is characterized by the following matrix

$$
\frac{\partial \Psi}{\partial z}=\left[\begin{array}{cccc}
1 & 0 & 0 & 0 \\
0 & 1 & 0 & 0 \\
0 & 0 & 1 & 0 \\
* & * & * & V_{X}-V_{H} z_{1}
\end{array}\right]
$$

Proposition 1 ([13]): The system (5) is observable if the rank of $\frac{\partial \Psi}{\partial z}$ is full, i.e. $\operatorname{Rank}\left(\frac{\partial \Psi}{\partial z}\right)=4$.

From the previous proposition, it yields that observability condition is fulfilled if

$$
V_{X}-V_{H} z_{1}>0
$$

In landing conditions, this observability condition is not respected when the aircraft is following the glide path. Indeed $z_{1}=\frac{V_{X}}{V_{H}}$ then the observability is lost. However, with an other choice of observability indicies, the observability condition is different. Then, as in [14][15], a solution consists in designing several observers and in switching between them according to observability loss. Nevertheless, this solution brings the difficulty of choosing the switching condition.

The proposed observer strategy, developed in the sequel, provides a smart solution to avoid switching. Consider the following transformation $\Psi$

$$
\zeta=\Psi(z)=\left[\begin{array}{llllll}
z_{1} & z_{2} & z_{3} & \dot{z}_{1} & \dot{z}_{2} & \dot{z}_{3}
\end{array}\right]^{T},
$$

Consider the following matrix $\frac{\partial \Psi}{\partial z}$ which will allow to characterize the capability to estimate $z_{4}$ from the measurement of $\boldsymbol{y}$

$$
\frac{\partial \Psi}{\partial z}=\left[\begin{array}{l}
\frac{\partial z_{1}{ }^{T}}{\partial z} \\
{\frac{\partial z_{2}}{\partial z}}^{T} \\
{\frac{\partial z_{3}}{\partial z}}^{T} \\
\frac{\partial \dot{z}_{1}}{\partial z^{T}} \\
\frac{\partial \dot{z}_{2}}{\partial z} \\
{ }^{T} \\
\frac{\partial \dot{z}_{2}}{\partial z}
\end{array}\right]=\left[\begin{array}{cccc}
1 & 0 & 0 & 0 \\
0 & 1 & 0 & 0 \\
0 & 0 & 1 & 0 \\
* & * & * & V_{X}-V_{H} z_{1} \\
* & * & * & V_{Y}-V_{H} z_{2} \\
* & * & * & V_{Y}-V_{H} z_{3}
\end{array}\right]
$$

with "*" terms that does not impact the observability.

From the previous proposition, it yields that observability condition is fulfilled if

$$
\left(V_{X}-V_{H} z_{1}\right)^{2}+\left(V_{Y}-V_{H} z_{2}\right)^{2}+\left(V_{Y}-V_{H} z_{3}\right)^{2}>0
$$

It appears that the observability condition (11) is fulfilled all along the landing phase. Actually, from (11), one can remark that, with $V_{H} \neq 0, V_{Y}-V_{H} z_{2}$ and $V_{Y}-V_{H} z_{3}$ could not be equal to zero at the same time. Indeed, $z_{2}=\frac{\Delta_{Y}-\frac{W}{2}}{\Delta_{H}}$ is different from $y_{3}=\frac{\Delta_{Y}+\frac{W}{2}}{\Delta_{H}}$. If $V_{H}=0$, the aircraft is flying "levelled", however $V_{X}$ is never null in fixed wing vehicle conventional landing. Then, the observability condition is always guaranteed for system (5).

\section{B. Observer design}

From (9) and (5), one gets

$$
\dot{\zeta}=\underbrace{\left[\begin{array}{llllll}
0 & 0 & 0 & 1 & 0 & 0 \\
0 & 0 & 0 & 0 & 1 & 0 \\
0 & 0 & 0 & 0 & 0 & 1 \\
0 & 0 & 0 & 0 & 0 & 0 \\
0 & 0 & 0 & 0 & 0 & 0 \\
0 & 0 & 0 & 0 & 0 & 0
\end{array}\right]}_{A}+\underbrace{\left[\begin{array}{c}
0 \\
0 \\
0 \\
\Phi_{1}(\mathbf{y}, \zeta) \\
\Phi_{2}(\mathbf{y}, \zeta) \\
\Phi_{3}(\mathbf{y}, \zeta)
\end{array}\right]}_{\Phi(\mathbf{y}, \zeta)}
$$

Proposition 2: An observer for system (12) reads as

$$
\dot{\hat{\zeta}}=A \hat{\zeta}+\Phi(\mathbf{y}, \hat{\zeta})+\kappa(\mathbf{y}, \hat{\zeta})
$$

with $\hat{\zeta}$ the estimated state of $\zeta$ and the function $\kappa(\mathbf{y}, \hat{\zeta})$ called "correction term" allowing to ensure $\hat{\zeta} \rightarrow \zeta$.

It is obvious that the correction term $\kappa(\mathbf{y}, \hat{\zeta})$ is not unique and can be obtained by several different methods depending on the desired features (robustness, finite time convergence, 
etc.,). Given that estimation error dynamics reads as (with $e=\hat{\zeta}-\zeta)$

$$
\dot{e}=A e+\Phi(\mathbf{y}, \hat{\zeta})-\Phi(\mathbf{y}, \zeta)+\kappa(y, \hat{\zeta}),
$$

$\kappa(\mathbf{y}, \hat{\zeta})$ has to make the observer converging to the real system in spite of the initial error $e(0)$. From $\hat{\zeta}=\Psi(\hat{z})$, one gets

$$
\dot{\hat{\zeta}}=\left[\frac{\partial \Psi}{\partial \hat{z}}\right] \dot{\hat{z}}
$$

The Jacobian matrix $\frac{\partial \Psi}{\partial \hat{z}}$ is not square. However, it is always left invertible because condition (11) is fulfilled all along the trajectory. So one can use its Moore-Penrose pseudo inverse ${ }^{1}$. One gets

$$
\dot{\hat{z}}=\left[\frac{\partial \Psi}{\partial \hat{z}}\right]^{+} \dot{\hat{\zeta}} .
$$

Proposition 3: If system (13) is an observer for the transformed system (12) then an observer for system (5) reads as

$$
\dot{\hat{z}}=f(\hat{z})+\left[\frac{\partial \Psi}{\partial \hat{z}}\right]^{+} \kappa(\mathbf{y}, \Psi(\hat{z}))
$$

Now, the function $\kappa(\mathbf{y}, \hat{z})$ has to be designed such that the estimate $\hat{z}$ converges to $z$. Two solutions are used in this paper.

High-Gain Observer [16]: The observer (17) for the system (5) admits a correction term $\kappa(\mathbf{y}, \hat{x})$ defined as

$$
\kappa(y, \hat{z})=\Lambda^{-1} K(\mathbf{y}-C \hat{z})
$$

with

$$
\Lambda=\left[\begin{array}{cccccc}
\lambda_{1} & 0 & 0 & 0 & 0 & 0 \\
0 & \lambda_{2} & 0 & 0 & 0 & 0 \\
0 & 0 & \lambda_{3} & 0 & 0 & 0 \\
0 & 0 & 0 & \lambda_{1}^{2} & 0 & 0 \\
0 & 0 & 0 & 0 & \lambda_{2}^{2} & 0 \\
0 & 0 & 0 & 0 & 0 & \lambda_{3}^{2}
\end{array}\right], \lambda_{i}>0
$$

and

$$
K=\left[\begin{array}{ccc}
K_{1} & 0 & 0 \\
0 & K 1 & 0 \\
0 & 0 & K 1 \\
K_{2} & 0 & 0 \\
0 & K_{2} & 0 \\
0 & 0 & K_{2}
\end{array}\right]
$$

with $K_{1}$ and $K_{2}$ strictly positive so that $A-K C$ is Hurwitz. The gains $K_{1}$ and $K_{2}$ are tuned in order to obtain estimation dynamics similar to a second order system with a damping coefficient $\xi$ and a pulsation $\omega$, which gives $K_{1}=2 \xi \omega$, $K_{2}=\omega^{2}$.

High-order sliding-mode observer [17]: An accurate and robust estimation of $\zeta$ is obtained by using highorder sliding-mode differentiation. Consider system (12) and

\footnotetext{
${ }^{1}$ with $M^{+}=\left(M^{T} M\right)^{-1} M^{T}$ the left pseudo inverse of a matrix M.
}

suppose that

H1: For $\zeta \in M_{\zeta}\left(M_{\zeta}\right.$ being the operating domain in $\zeta$-state space)

$$
\left|\Phi_{1}(\mathbf{y}, \zeta)\right|<L_{\Phi_{1}},\left|\Phi_{2}(\mathbf{y}, \zeta)\right|<L_{\Phi_{2}},\left|\Phi_{3}(\mathbf{y}, \zeta)\right|<L_{\Phi_{3}}
$$

with $L_{\Phi_{1}}, L_{\Phi_{2}}$ and $L_{\Phi_{3}}$ being known Lipschitz positive constants.

An observer based on high-order sliding-mode [17][18] for system (12) reads as

$$
\begin{aligned}
& \dot{\hat{\zeta}}_{1}=\hat{\zeta}_{4}+\underbrace{a_{1} L_{\Phi_{1}}^{\frac{1}{2}}\left|\zeta_{1}-\hat{\zeta}_{1}\right|^{\frac{1}{2}} \operatorname{sign}\left(\zeta_{1}-\hat{\zeta}_{1}\right)}_{\alpha_{1}} \\
& \dot{\hat{\zeta}}_{2}=\hat{\zeta}_{5}+\underbrace{a_{2} L_{\Phi_{2}}^{\frac{1}{2}}\left|\zeta_{2}-\hat{\zeta}_{2}\right|^{\frac{1}{2}} \operatorname{sign}\left(\zeta_{2}-\hat{\zeta}_{2}\right)}_{\alpha_{2}} \\
& \dot{\hat{\zeta}}_{3}=\hat{\zeta}_{6}+\underbrace{a_{3} L_{\Phi_{3}}^{\frac{1}{2}}\left|\zeta_{3}-\hat{\zeta}_{3}\right|^{\frac{1}{2}} \operatorname{sign}\left(\zeta_{3}-\hat{\zeta}_{3}\right)}_{\alpha_{3}} \\
& \dot{\hat{\zeta}}_{4}=\Phi_{1}(\mathbf{y}, \hat{\zeta})+a_{4} L_{\Phi_{1}} \operatorname{sign}\left(\alpha_{1}\right) \\
& \dot{\hat{\zeta}}_{5}=\Phi_{2}(\mathbf{y}, \hat{\zeta})+a_{5} L_{\Phi_{2}} \operatorname{sign}\left(\alpha_{2}\right) \\
& \dot{\hat{\zeta}}_{6}=\Phi_{3}(\mathbf{y}, \hat{\zeta})+a_{6} L_{\Phi_{3}} \operatorname{sign}\left(\alpha_{3}\right)
\end{aligned}
$$

Coefficients $a_{1}, a_{2}, a_{3}$ and $a_{4}$ must be fixed as proposed in [17]

$$
a_{1}=a_{2}=a_{3}=1.5, a_{4}=a_{5}=a_{6}=1.1 .
$$

Then, the observer (17) for the system (5) admits a correction term defined as

$$
\kappa(\mathbf{y}, \hat{z})=\left[\begin{array}{c}
\alpha_{1} \\
\alpha_{2} \\
\alpha_{3} \\
a_{2} L_{\Phi_{1}} \operatorname{sign}\left(\alpha_{1}\right) \\
a_{4} L_{\Phi_{2}} \operatorname{sign}\left(\alpha_{2}\right) \\
a_{6} L_{\Phi_{3}} \operatorname{sign}\left(\alpha_{3}\right)
\end{array}\right]
$$

by replacing in $\alpha_{i}, \zeta_{i}$ by $z_{i}, \hat{\zeta}_{i}$ by $\hat{z}_{i}$ (with $i \in\{1,2,3\}$ ).

\section{ApPliCATION TO A CIVIL AIRCRAFT LANDING}

\section{A. Landing scenarios}

Thanks to external systems, one can consider that the aircraft is reaching a zone close to the landing area. From these initial positions, camera sensor and vision scheme are used to estimate the deviations.

Precision of initial positions w.r.t. runway depends on several factor. For example, if GPS has been lost for a couple of hours, a Radio Navigation using IRS accelerometers integration coupled with $V O R-D M E$ system provides an absolute position impacted by a bias. Reduced navigation performance could also be caused by a lost of database or an unknown runway which induces that exact coordinates of the aiming point are not well-known. Nevertheless, bounded initial deviations of the aircraft w.r.t. the runway can be considered.

Three landing scenarios are proposed, each of them being characterized by three initial positions of the aircraft w.r.t. to the runway. Table I defines these three proposed initial 
positions; note that they correspond to an increasing difficulty (easy, medium, difficult) for landing scenario in term of dynamics of the aircraft.

\begin{tabular}{|c|c|c|c|c|c|c|}
\hline Landing & $\Delta_{X}(\mathrm{~m})$ & $\Delta_{Y}(\mathrm{~m})$ & $\Delta_{G S}(\mathrm{~m})$ & $\phi\left(^{\circ}\right)$ & $\gamma\left({ }^{\circ}\right)$ & $\Delta_{\psi}\left({ }^{\circ}\right)$ \\
\hline 1 & -5000 & +200 & -50 & 0 & -3 & 15 \\
\hline 2 & -5000 & -200 & +50 & 0 & -3 & 0 \\
\hline 3 & -4000 & -550 & +100 & 0 & -3 & -15 \\
\hline
\end{tabular}

TABLE I: Initial conditions for each landing scenario

Note that technological reasons lead to these initial values. Considering an on-the-shelf HD camera, initial value $\Delta_{X}=$ $-5000 m$ is an appropriate distance w.r.t. the runway to provide a sufficiently well-defined image for image processing purposes.

Based on civil aircraft actual navigation performances, the aircraft is able to reach a corridor containing the glide path; hence $\Delta_{Y}$ and $\Delta_{G S}$ are limited ${ }^{2}$. At the beginning of the scenarios, the aircraft is considered flying without roll angle $(\phi=0)$, with a flight path angle $\left(\gamma=-3^{\circ}\right)$ corresponding to the glide slope angle and with a heading difference $\Delta_{\psi}$, comprised between $\pm 15^{\circ}$.

Throughout this paper, three trajectories of the aircraft (see Figure 3), from the initial conditions of Table I, are used for the estimation study. These trajectories correspond to realistic

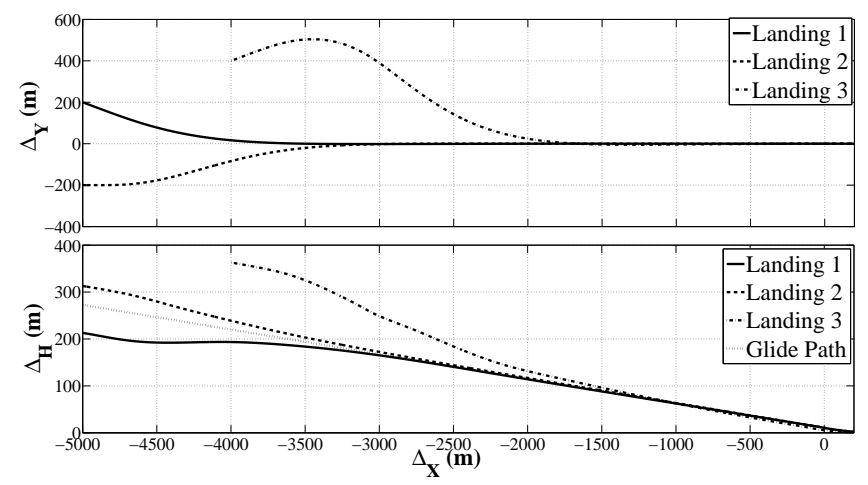

Fig. 3: Landings scenarios. Top. $\Delta_{Y}(m)$ versus $\Delta_{X}(m)$. Bottom. $\Delta_{H}(m)$ versus $\Delta_{X}(m)$.

behavior of an aircraft in landing conditions. Note that the aircraft presents a saturated vertical behavior $(\gamma>-6)$ in Landing 3 which leads to a poorer glide tracking than other landings.

\section{Assumptions}

- Looking the behavior of the aircraft and by an adequate choice of camera (in term of field-of-view), the runway can be considered in the image all along the landings.

- During landing, the speed of the aircraft is supposed to be maintained around the approach speed value (here 140 knots) thanks to an independent controller.

- Visibility is assumed to be good enough. Hence visible frequency range is used. However, the concept could

\footnotetext{
${ }^{2}$ e.g. $\left|\Delta_{Y}\right|<200 m$ with a $68.3 \%$ probability and $\left|\Delta_{Y}\right|<550 \mathrm{~m}$ with $99.7 \%$ and $\left|\Delta_{G S}\right|<100 m$ thanks to barometers or radio-altimeter sensor
}

be extended to other type of sensors (e.g. Infra-Red or millimeter-wave camera).

\section{B. Simulation results}

The previous observers have been evaluated on each landing scenarios. The estimated variables are initialized with a rough value corresponding to an error of $20 \%$ w.r.t. the deviations. The high gain correction term is designed with damping coefficient $\xi=0.7$ and pulsation $\omega=1$ corresponding to a response time of $5 s$. Terms in (19) are taken at $\lambda_{i}=1$. A $1 \%$ random noise is added on the measured signals which corresponds to an image processing noise. Results obtained along the three defined landings in presence of noisy measurement for a high gain observer are presented in Figure 4.

It appears that the high gain observer presents a good
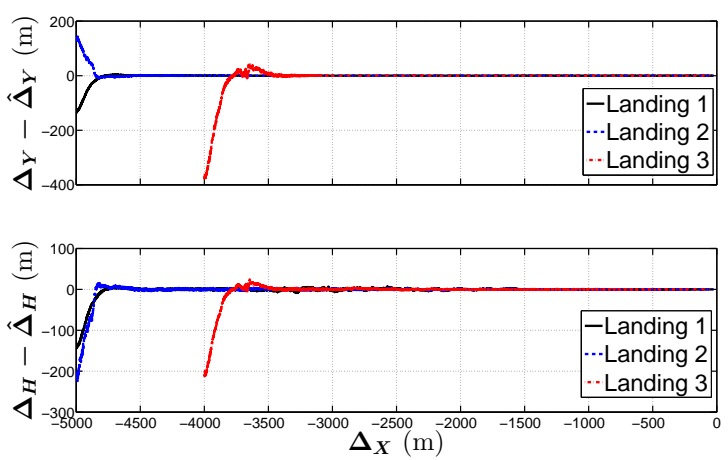

Fig. 4: High gain observer. Top. Estimation error of $\left(\Delta_{Y}(m)\right)$ with respect to $\Delta_{X}(m)$, for the three landing scenarios in presence of noisy measurements.Bottom. Estimation error of $\Delta_{H}(m)$ with respect to $\Delta_{X}(m)$, for the three landing scenarios in presence of noisy measurements.

behavior all along the 3 landings scenarios. Indeed, the estimation error presented in Figure 4 converges to zero with a fast dynamics compared with the dynamics of the aircraft.

A similar dynamics of the observation with a correction term based on high order sliding-mode observer is designed. It corresponds to a parameter tunning such that $\alpha_{1}=1.5$, $\alpha_{2}=1.1$ as proposed in [17] and $L_{1}=0.0005$. Results obtained along the three defined landings in presence of noisy measurement for an high order sliding-mode observer are presented in Figure 5.

The high order sliding-mode observer presents also a good behavior in terms of convergence and dynamics. Nevertheless, the noisy measurement has more effects on the estimation results than with the high gain observer. Actually, sliding-mode is well known for a chattering effect which is here reduced thanks to the high order of the solution. Moreover, one can remark that the worst estimation arises when $\Delta_{X}$ is between $-3500 m$ and $-2000 m$ only for Landing 1 and Landing 2. For the end of the landing, these effects disappears. This behavior could be explained because 

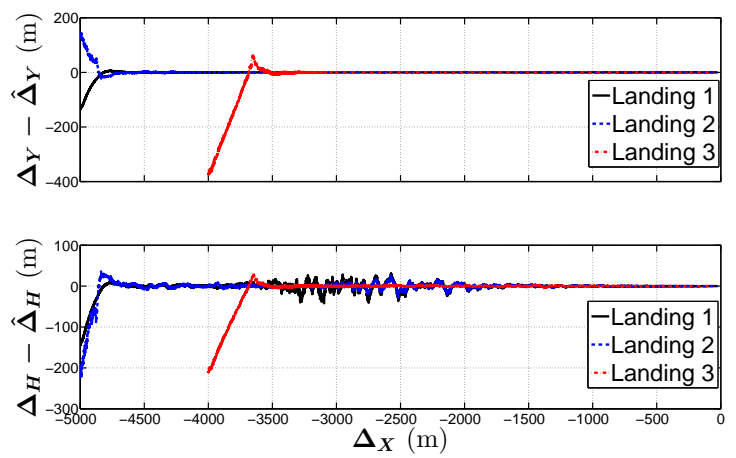

Fig. 5: High order sliding-mode observer. Top. Estimation error of $\left(\Delta_{Y}(m)\right)$ with respect to $\Delta_{X}(m)$, for the three landing scenarios in presence of noisy measurements. Bottom. Estimation error of $\Delta_{H}(m)$ with respect to $\Delta_{X}(m)$, for the three landing scenarios in presence of noisy measurements.

of the important range between the aircraft and the runway compared with the runway width. When the aircraft is aligned, the observability feature decreases which produces oscillations due to noisy measurements. Then, when the aircraft is coming closer to the runway, observability feature is increasing which reduces the effect of noise. Note that Landing 3 estimation results, the aircraft is aligned later than for Landing 1 and 2. Then, noise does not impact the estimation for this landing.

To conclude, the proposed estimation solution provides the ability to easily design different types of observers as high-gain or high-order sliding-mode. The observers provides a good estimation of the deviation with a good convergence rate and no static error. The robustness to noisy measurement depends on the observability condition. The proposed solution uses only the first two corners of the runway which guaranties observability feature but by using other visual features as for example the ending corners of the runway, observability could be improved and should provide robustness to noisy measurement.

\section{CONCLUSION AND FUture PERSPECTIVES}

This paper proposes a relative pose estimation solution for automatic landings of civil aircraft with a reduced number of informations coming from external means. Thanks to an adequate coordinates transformation, a frame for designing an observer is proposed. This ensure performance despite the potential loss of observability. Nonlinear observer (high-gain and high-order sliding-mode) have been applied in order to get robust and online estimation on several landing scenarios. Future works will focus on the effect of estimation in guidance performances. Noise, calibrations errors and delay caused by Image Processing will be explored. Moreover, a complete landing estimation solution will be performed to handle the problem of losing the corners of the runway in the image or to keep them in the image as in [19].

\section{ACKNOWLEDGMENT}

The present research is partially supported by ANR VISIOLAND project ANR-13-CORD-0012 dedicated to VISIOnbased aircraft LANDing techniques.

\section{REFERENCES}

[1] F. Chaumette and S. Hutchinson, "Visual servo control. i. basic approaches," Robotics Automation Magazine, IEEE, vol. 13, no. 4, pp. 82-90, 2006.

[2] J. R. Azinheira and P. Rives, "Image-Based Visual Servoing for Vanishing Features and Ground Lines Tracking: Application to a UAV Automatic Landing," International Journal of Optomechatronics, vol. 2, pp. 275-295, 2008.

[3] L. Coutard, F. Chaumette, and J.-M. Pflimlin, "Automatic landing on aircraft carrier by visual servoing," in Intelligent Robots and Systems (IROS), 2011 IEEE/RSJ International Conference on, 2011, pp. 28432848.

[4] F. Le Bras, T. Hamel, C. Barat, and R. Mahony, "Nonlinear imagebased visual servo controller for automatic landing guidance of a fixedwing aircraft," in European Control Conference, Budapest, Hungary, 2009.

[5] V. Gibert and G. Puyou, "Landing of a transport aircraft using image based visual servoing," in 9th IFAC Symposium on Nonlinear Control Systems (NOLCOS), Toulouse, France, 2013.

[6] L. Burlion, H. de Plinval, and P. Mouyon, "Backstepping based visual servoing for transport aircraft automatic landing," in IEEE Conference on Control Application (CCA), Oct 2014.

[7] P. Trisiripisal, M. R. Parks, A. L. Abbott, T. Liu, and G. A. Fleming, "Stereo analysis for vision-based guidance and control of aircraft landing," in 44th AIAA Aerospace Science Meeting and Exhibit, Reno, Nevada, USA, 2006.

[8] Y. Gui, P. Guo, H. Zhang, Z. Lei, X. Zhou, J. Du, and Q. Yu, "Airborne vision-based navigation method for uav accuracy landing using infrared lamps," Journal of Intelligent \& Robotic Systems, vol. 72, no. 2, pp. 197-218, 2013.

[9] O. Dahl, F. Nyberg, J. Holst, and A. Heyden, "Linear design of a nonlinear observer for perspective systems," in IEEE International Conference on Robotics and Automation (ICRA), Barcelona, Spain, 2005.

[10] P. Giordano, A. De Luca, and G. Oriolo, "3d structure identification from image moments," in IEEE International Conference on Robotics and Automation, Pasadena, California, USA, 2008, pp. 93-100.

[11] D. Karagiannis and A. Astolfi, "A new solution to the problem of range identification in perspective vision systems," IEEE Transactions on Automatic Control, vol. 50, no. 12, pp. 2074-2077, 2005.

[12] X. Chen and H. Kano, "A new state observer for perspective systems," IEEE Transactions on Automatic Control, vol. 47, no. 4, pp. 658-663, 2002.

[13] A. J. Krener and W. Respondek, "Nonlinear observers with linearizable error dynamics," SIAM Journal on Control and Optimization, vol. 23, no. 2, pp. 197-216, 1985.

[14] V. Lebastard, Y. Aoustin, and F. Plestan, "Estimation of absolute orientation for a bipedal robot: Experimental results," Robotics, IEEE Transactions on, vol. 27, no. 1, pp. 170-174, 2011.

[15] C. El Tannoury, S. Moussaoui, F. Plestan, N. Romani, and G. Pita-Gil, "Synthesis and application of nonlinear observers for the estimation of tire effective radius and rolling resistance of an automotive vehicle," Control Systems Technology, IEEE Transactions on, vol. 21, no. 6, pp. 2408-2416, Nov 2013.

[16] J. P. Gauthier, H. Hammouri, and S. Othman, "A simple observer for nonlinear systems, application to bioreactors," IEEE Transactions on Automatic Control, vol. 37, no. 6, pp. 875-880, 1992.

[17] A. Levant, "Higher-order sliding modes, differentiation and outputfeedback control," International Journal of Control, vol. 76, no. 9-10, pp. 924-941, 2003.

[18] - ,Finite-time stability and high relative degrees in sliding-mode control," in Sliding Modes after the First Decade of the 21st Century, ser. Lecture Notes in Control and Information Sciences, L. Fridman, J. Moreno, and R. Iriarte, Eds. Springer Berlin Heidelberg, 2012, vol. 412, pp. 59-92.

[19] L. Burlion and H. de Plinval, "Keeping a ground point in the camera field of view of a landing uav," in IEEE International Conference on Robotics and Automation (ICRA), May 2013. 